\title{
Metallic Periodic Surface Lattice Enhanced High-Power MM-wave Sources
}

\author{
A.J. MacLachlan, H. Yin, L. Zhang, C.R. Robertson, K. Ronald, A.W. Cross, and A.D.R. Phelps \\ Department of Physics, SUPA, University of Strathclyde, Glasgow, G4 0NG, Scotland, UK
}

\begin{abstract}
The design and construction of metallic Periodic Surface Lattices (PSLs) to enable oversized cylindrical interaction volumes to be excited efficiently by annular electron beams is presented. Construction methods include metallic electrodeposition and "additive manufacturing", or "3D printing".
\end{abstract}

\section{INTRODUCTION}

$\mathrm{T}$ $\mathrm{O}$ increase the potential power output of an electron beam electromagnetic wave interaction, either the applied accelerating voltage of the electron beam should be increased and/or the beam current increased. Our interest is in creating high power electron beam driven mm-wave sources. Historically as the source frequency has increased and the free-space wavelength has decreased the interaction structures have needed to become correspondingly smaller in order to achieve an efficient interaction by limiting the number of modes within the interaction structure. Electron beam current density cannot be arbitrarily increased however as at some point the internal space charge forces on the beam, and the influence of the surrounding environment contrive to impose limits on the beam stability and propagation. In addition, the increased RF power density can result in breakdown and the effect known as 'pulse shortening'. To avoid these issues, it is necessary to consider the possibility of increasing the potential power output by increasing the transverse size of the interaction structure and of the electron beam, thereby achieving higher currents without excessively high current densities. Unfortunately, increasing the transverse size of the interaction region beyond a ratio of $\mathrm{D} / \lambda \sim 2$ (where $\mathrm{D}$ is the transverse diameter of the electromagnetic interaction structure and $\lambda$ is the radiation wavelength) results in a drop in efficiency and spectral purity as the source loses mode selectivity.

To solve this problem, the use of a metallic two dimensional periodic surface lattice (PSL) [1-5] to achieve a coupling of 4 waves (a forward and backward longitudinal wave and a clockwise and anti-clockwise azimuthal wave) to control the transverse modes which can interact with the electron beam in an oversized interaction space has been proposed. This potentially enables reasonably high efficiency even when the ratio of the transverse diameter of the interaction structure to the radiation wavelength $D / \lambda \geq 4$ and eventually higher values of $\mathrm{D} / \lambda \geq 10$ may become feasible.

The cylindrical 2D PSL structure can be described by equation (1)

$$
r=r_{0}+\Delta r \cos \left(k_{z} z\right) \cos (m \emptyset)
$$

where $r_{0}$ is the radius of the unperturbed waveguide, $\Delta r$ is the amplitude of the corrugation, $k_{z}=2 \pi / d_{z}$, where $d_{z}$ is the period of the corrugation over the $\mathrm{z}$ - coordinate and $m$ is the number of azimuthal variations of the corrugation.

In an earlier experiment "additive manufacturing" [6] or "3D printing" was used to construct a silver alloy $2 \mathrm{D}$ periodic surface lattice structure that was designed to be incorporated in a W-band source $(75-110 \mathrm{GHz})$. 3D printing developed in the mid 1980's and the number of its applications is continuing to expand. The tolerances achievable with 3D printing are projected to improve over time as the technology develops, resulting in higher degrees of accuracy. The operation of this W-band mm-wave source using the 3D printed PSL has been reported [7]. This method of construction of interaction structures has also been successfully demonstrated in a high power microwave source by French and Shiffler [8].

\section{RESULTS}

The parameters $r_{0}, \Delta r, d_{z}, m$ and the number of longitudinal periods $n$ have been calculated to design several optimized PSLs. The optimum value of $d_{z}$ is determined by the magnitude of the longitudinal component of the electron beam velocity and the desired mm-wave output frequency. In our present work, following the optimization of the PSL design, the PSL construction by an electrodeposition method Fig. 1(a) has been compared with a 3D printing method Fig. 1(b).

The electrodeposition method is a conventional method frequently used in making microwave and millimeter-wave structures. It starts with the machining of a positive mandrel of aluminium, upon which copper is grown by electrodeposition. The aluminium is then dissolved away using caustic soda leaving the desired cylindrical cavity structure.

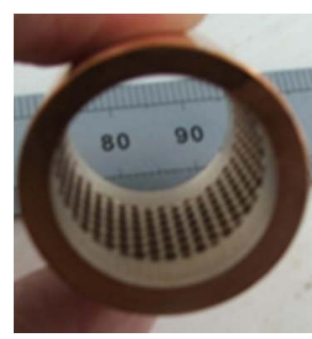

(a)

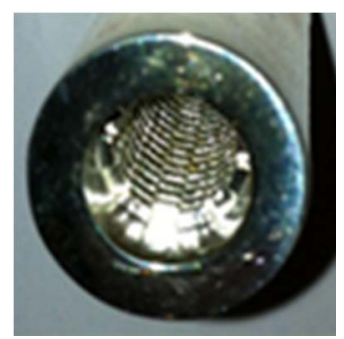

(b)
Fig. 1 Examples of metallic periodic surface lattice enhanced cavities (a) copper cavity constructed using electrodeposition on an aluminium mandrel (b) silver/chromium alloy cavity manufactured by a casting process using a $3 \mathrm{D}$ printed pattern to create the mold.

There are several methods of manufacturing the cylindrical cavity structures using $3 \mathrm{D}$ printing, including direct printing in metal. In our case the 3D printing procedure is a two stage process. The first stage involves creating a wax former to the 10 's of microns precision and then using this former to create a mold for the component, where the silver - chromium molten alloy is deposited. The transmission and reflection 
properties of the cylindrical PSL structures are measured, as a function of frequency, using a Vector Network Analyser.

Compared with the previous experiment [7], improvements have been made to the electron gun and the solenoidal magnetic field system used to transport the annular electron beam through the cylindrical PSL interaction structure. An annular graphite cathode, with a sharpened annular emitting tip is used to create a hollow annular electron beam [9]. This annular electron beam is guided through the interaction cavity using the applied axial magnetic field and is designed to pass close to the inner surface of the cylindrical periodic surface lattice. In the experiments reported in 2017 [7] the magnetic field increased along the axis from the location of the electron emission from the cathode surface to the interaction region inside the cavity, resulting in a higher value of $\alpha$ (the ratio of the perpendicular component of the electron velocity to the parallel component of the electron velocity) than desirable. Since this is designed to be a slow wave Cherenkov-type of interaction, rather than a fast wave interaction based on the electron cyclotron gain mechanism used in gyro-devices, a near to zero value of the component of the electron velocity perpendicular to the axial magnetic is preferable. In the new experiments, that are ongoing in 2018, the magnitude of the axial guiding magnetic field is maintained constant as a function of the axial position from the location of the electron emission on the cathode surface through to the interaction region inside the cavity. The value of $\alpha$ is designed to be as near zero as practically possible at the cathode and so with the guiding magnetic field being held constant axially the resultant value of $\alpha$ in the region inside the interaction cavity is near to zero and significantly smaller than in the earlier experiments. Faraday cup, witness plate and current/voltage diagnostics are used to measure the electron beam.

In the earlier reported experiments [7], cut-off filter measurements were used to give an estimate of the mm-wave output frequency. However a more accurate frequency measurement using a heterodyne frequency diagnostic is being used in the new series of experiments during 2018 to be able to more definitively demonstrate that the cylindrical PSL enables successful coupling between the surface and volume fields.

\section{SUMMARY}

The design and construction of optimized metallic cylindrical PSLs and the use of these PSLs in an experimental study of an electron beam driven W-band enhanced mm-wave source are reported. In future work, using similar principles to those used in the W-band experiments it is proposed to extend the working frequency of these metallic periodic surface lattice enhanced high-power sources to $200 \mathrm{GHz}$ and beyond.

\section{ACKNOWLEDGMENTS}

This work has been supported by the Leverhulme Trust, UK, International Network Grant IN-2015-012 and by the AFOSR award numbers FA8655-13-1-2132 and FA9550-17-1-0095.

\section{REFERENCES}

[1]. A. W. Cross, I. V. Konoplev, A. D. R. Phelps, et al., "Studies of surface two-dimensional photonic band-gap structures", J. Appl. Phys., vol. 93, pp. 2208-2218, Feb., 2003.
[2]. I. V. Konoplev, L. Fisher, K. Ronald, A. W. Cross, A. D. R. Phelps, C. W. Robertson, and M. Thumm, "Surface-field cavity based on a two-dimensional cylindrical lattice," Appl. Phys. Lett., vol. 96, art. no. 231111, June, 2010.

[3]. I. V. Konoplev, L. Fisher, A. W. Cross, A. D. R. Phelps, K. Ronald, and C. W. Robertson, "Surface wave Cherenkov maser based on a periodic lattice," Appl. Phys. Lett., vol. 96, art. no. 261101, June, 2010.

[4]. I. V. Konoplev, L. Fisher, A. W. Cross, A. D. R. Phelps, K. Ronald, and M. Thumm, "Excitation of surface field cavity and coherence of electromagnetic field scattering on two-dimensional cylindrical lattice," Appl. Phys. Lett, vol. 97, art. no. 261102, Dec., 2010.

[5]. I. V. Konoplev, A. J. MacLachlan, C. W. Robertson, et al., "Cylindrical, periodic surface lattice - Theory, dispersion analysis, and experiment," Appl. Phys. Lett., vol. 101, art. no. 121111, Sept., 2012.

[6]. I. Gibson, D. Rosen and B. Stucker, "Additive Manufacturing Technologies: $3 D$ Printing, Rapid Prototyping, and Direct Digital Manufacturing", 2nd Edition, Springer, New York, USA, 2015.

[7]. A. R. Phipps, A. J. MacLachlan, C. W. Robertson, et al., "Electron beam excitation of coherent sub-terahertz radiation in periodic structures manufactured by 3D printing," Nucl. Instrum. Methods Phys. Res. B, vol. 402, pp. 202-205, July, 2017.

[8]. D. M. French and D. Shiffler, "High power microwave source with a three dimensional printed metamaterial slow-wave structure", Rev. Sci. Instrum., vol. 87, art. no. 053308, May, 2016.

[9]. I. V. Konoplev, A. W. Cross, P. MacInnes, et al., "High-current oversized annular electron beam formation for high-power microwave research," Appl. Phys. Lett., vol. 89, art. no. 171503, Oct., 2006. 